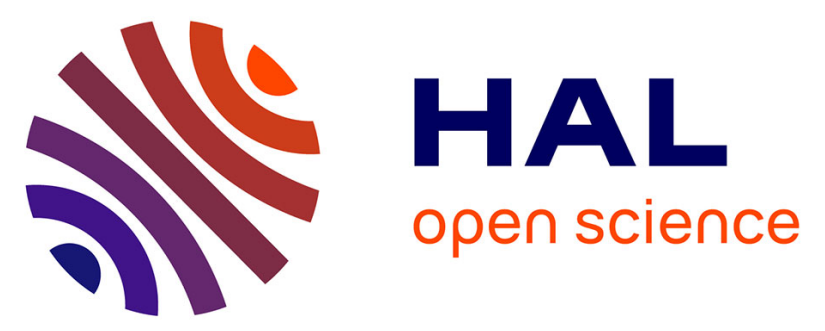

\title{
Multi-physics model of an electric fish-like robot: numerical aspects and application to obstacle avoidance
}

Mathieu Porez, Vincent Lebastard, Auke Jan Ijspeert, Frédéric Boyer

\section{To cite this version:}

Mathieu Porez, Vincent Lebastard, Auke Jan Ijspeert, Frédéric Boyer. Multi-physics model of an electric fish-like robot: numerical aspects and application to obstacle avoidance. IEEE/RSJ International Conference on Intelligent Robots and Systems, Sep 2011, San-Francisco, United States. pp.1-6. hal-00630762

\section{HAL Id: hal-00630762 https://hal.science/hal-00630762}

Submitted on 11 Oct 2011

HAL is a multi-disciplinary open access archive for the deposit and dissemination of scientific research documents, whether they are published or not. The documents may come from teaching and research institutions in France or abroad, or from public or private research centers.
L'archive ouverte pluridisciplinaire HAL, est destinée au dépôt et à la diffusion de documents scientifiques de niveau recherche, publiés ou non, émanant des établissements d'enseignement et de recherche français ou étrangers, des laboratoires publics ou privés. 


\title{
Multi-physics model of an electric fish-like robot : numerical aspects and application to obstacle avoidance
}

\author{
Mathieu POREZ, Vincent LEBASTARD, Auke Jan IJSPEERT and Frédéric BOYER .
}

\begin{abstract}
The paper deals with the modeling of a fishlike robot equipped with the electric sense, suited to study sensorimotor loops. The proposed multi-physics model merges a swimming dynamic model of a fish-like robot with an electric model of an embedded electrolocation sensor. Based on a TCPIP and threaded framework, the resulting simulator works in real time. After presenting the modeling aspects of this work, this article focuses on two numerical studies. In the first, the interactions between body deformations and perception variables are studied and a current correction process is proposed. In the second study, an electric exteroceptive feedback loop based on a direct current measurement method is designed and tested for obstacle avoidance.
\end{abstract}

\section{INTRODUCTION}

Lissmann in the 1950's [1] was among the first scientists to clearly demonstrate the electric nature of the perception of the weakly electric fish. He assessed that "the electric organ discharges belong to a full sensorial system and are used for scanning the environment and for the interactions with the other electric fishes". After this discovery, scientists begun to study in detail how the environment was electrically interpreted by the electric fish. Brian Rasnow in 1996 [2] developed the first model of interactions between the environment and the electric currents flowing through the skin of the fish, that we call the electric image. His model derived from simple electromagnetism conditions is dedicated to the study of the effects of a sphere placed in the vicinity of the fish. His simple model showed the relation between the shape of the electric image and the distance and dimensions of spherical objects. Recently Solberg \& al [3] have designed a robotic detection device based on the electric sense. They performed with their device an automatic detection of a sphere. In its current form, it seems that their device is more suited for the design of perception algorithms rather than for an implementation on an existing autonomous robot. More recently, a new project was started in Europe: ANGELS ${ }^{1}$ (for ANGuilliform robot with ELectric Sense). Its objective is to build an eel-like robot equipped with an electric sensor. The ANGELS' robot would be capable to navigate using the electric sense and to divide itself in several autonomous individual modules for exploration.

M. POREZ and A. J. IJSPEERT are with BioRob, EPFL STI - IBI - BIOROB, Station 14, 1015 Lausanne, Switzerland. mathieu.porez@epfl.ch and auke.ijspeerteepfl.ch Vincent LEBASTARD and Frédéric BOYER are with IRCCyN, EMN - La Chantrerie - 4, rue Alfred Kastler,B.P. 20722, 44307 Nantes Cedex 3 France. vincent. lebastard@mines-nantes. fr and frederic.boyeremines-nantes. fr

${ }^{1} \mathrm{http}: / / \mathrm{www}$.theangelsproject.eu/
In the ANGELS' context, the article deals with the actionperception loops. In the case of an electric fish-like robot, this problem consists in deriving the laws that rule: $1^{\circ}$ ) the fish and fluid dynamics; $2^{\circ}$ ) electromagnetic phenomena; $3^{\circ}$ ) sensorimotor feedback; involved by the locomotion, perception and their couplings. Thus, in this paper, we propose a model and a simulator in order to study the action-perception problem.

The article is structured as follows. The modeling of an electric fish-like robot is first presented in section II. In section III, the models are implemented in a modular simulation architecture. Then, the resulting simulator is exploited for obstacle avoidance in section IV in the case of the AmphiBot robot of BioRob Lab ${ }^{2}$. Lastly, the article ends with section $\mathrm{V}$ by some concluding remarks.

\section{THE ELECTRIC FISH MODELING}

\section{A. The problem statement}

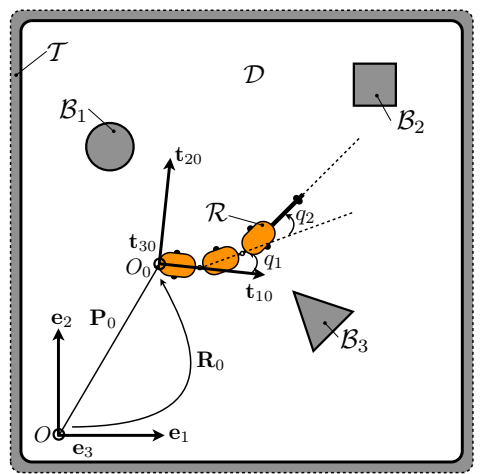

Fig. 1. The problem statement.

Before developing the different modeling and numerical aspects of this work, let us define the problem statement. As illustrated in Fig.1, let us consider a fish-like robot (denoted by $\mathcal{R}$ ) swimming in an insulating tank (denoted by $\mathcal{T}$ ) including $L$ objects (denoted by $\mathcal{B}_{k}$, where $k$ denotes the body index). We define by $\mathcal{D}=\mathcal{T}-\mathcal{R}-\sum_{k=1}^{L} \mathcal{B}_{k}$ the sub-domain containing at any time the homogeneous Ohmic fluid of $\gamma$ conductivity. Moreover, the location of any point of $\mathcal{D}$ is defined by the position vector $\boldsymbol{x}=x_{j} \boldsymbol{e}_{j}$ with respect to the Galilean frame $\mathcal{F}_{g}=\left(O, \boldsymbol{e}_{1}, \boldsymbol{e}_{2}, \boldsymbol{e}_{3}\right)$. The fish-like robot is a two-dimensional swimmer, which is composed of $N$ actuated modules, a head and a caudal fin. At any time $t$, the robot configuration is defined by the

\footnotetext{
${ }^{2}$ http://biorob.epfl.ch/
} 
joint positions $q$ together with the orientation matrix $\boldsymbol{R}_{0}$ and the position vector $\boldsymbol{P}_{0}$ of a mobile frame attached to the robot head $\mathcal{F}_{0}=\left(O, \boldsymbol{t}_{1}, \boldsymbol{t}_{2}, \boldsymbol{t}_{3}\right)$ with respect to $\mathcal{F}_{g}$. Finally, the robot is equipped by discharge electrodes on the tail and measurement electrodes placed on its head in order to electro-sense its environment. In the following, the electrodes are denoted by $\epsilon_{i}$ (where $i$ denotes the electrode index).

On the base of this statement, to study the actionperception interactions, we must model: $1^{\circ}$ ) the swimming dynamics of a self-propelled fish-like robot. $2^{\circ}$ ) the electric currents crossing the robot's electrodes in function of the electric activity of its discharge electrodes and the environment; $3^{\circ}$ ) the sensorimotor feedback coupling the current measurements with the parameters of the anguilliform swimming gaits or transient maneuvers. Let us now detail each of them.

\section{B. The swimming modeling}

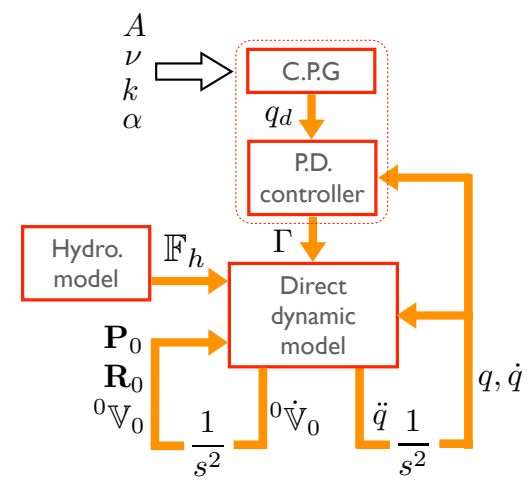

Fig. 2. Schematic view of the swimming model.

In the general case, the swimming problem consists in the following sequence of physical causes and effects: $1^{\circ}$ ) in order to move, any swimming robot actuates its internal (shape) degrees of freedom; $2^{\circ}$ ) then, the new body shape disturbs the media which generates contact forces on the robot's skin; $3^{\circ}$ ) finally, these forces trust the external degrees of freedom of the head module. In this string of causalities : the following coupled dynamics appear: the external dynamics for the locomotion, the media dynamics for the contact forces and the internal dynamics for the locomotion control. To model properly a fish-like robot, we must resolve these coupled dynamics. For this purpose, we propose to exploit the three following recent contributions on this topic: $1^{\circ}$ ) the Central Pattern Generator (CPG) by Crespi \& al [4] for the locomotion control; $2^{\circ}$ ) the recursive algorithms based on the Newton-Euler (N-E)'s equations by Khalil \& al [5] for the robot dynamic modeling; $3^{\circ}$ ) the analytical hydrodynamic model of a 3-D self-propelled fish swimming by Boyer \& al [6], [7] for the hydrodynamic force modeling. As shown on Fig.2, the proposed swimming model is composed by: $1^{\circ}$ ) a locomotion controller; $2^{\circ}$ ) a direct dynamic model; $3^{\circ}$ ) a hydrodynamic model.

1) The locomotion controller: It is composed of a CPG and a proportional derivative PD controller computing, re- spectively, the joint set points $q_{d}$ and the torques $\Gamma$ applied by the motors on joints. A simple manner to generate the swimming rhythmic motions of fishes (see [8]) is to use a CPG, i.e. a system of coupled nonlinear oscillators inspired from the locomotor circuits found in the spinal cord of vertebrates (e.g. the lamprey). This bio-inspired controller has several explicit parameters, which can be continuously modified, controlling the body undulation shape for forward and backward swimming (through the wave amplitude $A$, the wave frequency $\nu$ and the number of waves along the robot backbone $k$ ) and the average curvature for turning maneuvers (through the backbone average curvature $\alpha$ ). In addition to its reduced parameter set, the CPG can adapt quickly and smoothly the joint trajectories after any abrupt parameter change. In this paper, this last property will be extensively used to smoothly modulate the swimming gaits depending on the electro-sense variables.

2) The direct dynamic model: In this work, the computing of the swimming dynamics of the fish-like robot is achieved by the direct recursive N-E algorithm developed in [5]. The algorithm based on Newton's law and Euler's theorem is dedicated to the dynamic modeling of mobile serial robots. It allows to compute, as a function of $\Gamma$, the robot motion, i.e. the robot head accelerations ${ }^{0} \dot{\mathbb{V}}_{0}$ with respect to $\mathcal{F}_{g}$ together with the joint accelerations $\ddot{q}$. At each step of a global time integration loop; the direct algorithm solves one after one the three following recursive loops dedicated to : $1^{\circ}$ ) the robot kinematics; $2^{\circ}$ ) the external dynamics taking into account the hydrodynamic forces $\mathbb{F}_{h} ; 3^{\circ}$ ) the internal dynamics.

3) The hydrodynamic model: As far as the hydrodynamic is concerned, we used here the generalization of the large amplitude elongated body theory of Lighthill (see [9]) proposed in [7] and numerically validated in [6]. This model appears as a superimposition of a reactive model with a resistive one which depends only on segment motions. As regards the reactive part of the hydrodynamic model, the thrust and the lateral lift are modeled through the effect of the fluid inertia on the undulating fish body. Basically, it is based on the slender body theory of Munk [10], where the 3-D flow around an elongated body is approximated by a stratification of planar lateral flows, which are then resolved analytically. Concerning the resistive part, the effect of the fluid viscosity on the nose and on the skin of the fish is modeling through a Taylor-like resistive model [11].

\section{The electric modeling}

From a technical view point, the working of the electricperception sensor embedded in the robot is based on the generation of an electric field $\boldsymbol{E}$ in the water by polarization of measurement electrodes with respect to discharge ones. The robot body having a lower conductivity than the water, hence $\boldsymbol{E}$ is focalized through the measurement electrodes. By this mechanism, the robot can build an electric image of its environment by comparison between the expected currents (measured in a free environment) and those actually measured. From the point of view of electromagnetism, the electric state of the fluid can be considered as quasi steady. 
Thus the electric field is irrotational and can be determined by the gradient of the electric potential field $\phi$. Then, the Ohm law and the conservation of electric currents allow one to state the set of partial differential equations $\Delta \phi=0$ named Laplace equations, which rule $\phi \in \mathcal{D}$ (hence $\boldsymbol{E}=$ $-\nabla \phi)$ with respect to the electric conditions imposed on $\partial \mathcal{D}$ (i.e. the electrodes, robot body, tank and passive objects). In order to resolve the Laplace equations, we propose to use the 2-D Boundary Integral Equations (BIE) formulation and its discretization using the conventional Boundary Element Method (BEM) [12]. The solution to the boundary value problem described by the Laplace equations can be written for any internal point $\boldsymbol{x}_{0} \in \mathcal{D}$ by:

$$
\phi\left(\boldsymbol{x}_{0}\right)=\int_{\partial \mathcal{D}} G\left(\boldsymbol{x}_{0}, \boldsymbol{x}\right) E_{\perp}^{+}(\boldsymbol{x}) d l-\int_{\partial \mathcal{D}} F\left(\boldsymbol{x}_{0}, \boldsymbol{x}\right) \phi(\boldsymbol{x}) d l,
$$

where $E(\boldsymbol{x})_{\perp}^{+}=(\partial \phi / \partial n)(\boldsymbol{x})$ (with $\boldsymbol{x} \in \partial \mathcal{D}$ and $\boldsymbol{n}$ the outward normal to $\partial \mathcal{D}), F=\left(\partial G\left(\boldsymbol{x}_{0}, \boldsymbol{x}\right) / \partial n\right)(\boldsymbol{x})$ and $G\left(\boldsymbol{x}_{0}, \boldsymbol{x}\right)$ are the Green's function in 2-D space define by:

$$
G\left(\boldsymbol{x}_{0}, \boldsymbol{x}\right)=\frac{1}{2 \pi} \ln \left(\frac{1}{r}\right) \text { and } F\left(\boldsymbol{x}_{0}, \boldsymbol{x}\right)=\frac{1}{2 \pi r} \frac{\partial r}{\partial n},
$$

where $r=\left\|\boldsymbol{x}-\boldsymbol{x}_{0}\right\|$. In order to numerically solve (1), the BEM consists in meshing $\partial \mathcal{D}$ into $M$ boundary elements $\mathcal{L}_{l}$, i.e. $\partial \mathcal{D}=\bigcup_{l=1}^{M} \mathcal{L}_{l}$ (see Fig.3). Then, the two layers of singularities $\phi$ and $E_{\perp}^{+}$are approximated on each elements through a polynomial interpolation of their nodal values. Hence, the electric potential $\phi_{l}$ computing in $\boldsymbol{x}_{\boldsymbol{l}}$, center of the $l^{t h}$ element, can be written as the discrete superimposition of the contributions due to the elements $m=1, \ldots M$, i.e.

$$
\frac{1}{2} \phi_{l}=\sum_{j=1}^{M} E_{\perp m}^{+} \int_{\mathcal{L}_{m}} \ln \left(\frac{1}{r}\right) d l-\sum_{m=1 \neq l}^{M} \phi_{m} \int_{\mathcal{L}_{m}} \frac{1}{r} \frac{\partial r}{\partial n_{m}} d l .
$$

Then, the $M$ equations (2) can be written in the form of an implicit linear system, in applying the boundary conditions to $\phi$ and $E_{\perp}^{+}$. After the resolution of (2), the currents $I_{i}$ crossing electrodes $\epsilon_{i}$ are computed as follows: $I_{i}=\gamma \eta \sum E_{\perp l}^{+} L_{l}$ where $\eta$ is a 2-D/3-D correlation coefficient and $L_{l}$ is the length of the element $\mathcal{L}_{l}$. Let us note that the last sum operation is realized only on constituent meshes of $\epsilon_{i}$. Finally, we realized an experimental validation of our numerical model. The used set-up is described in [14]. The differences between the currents given by our 2-D BEM solver and experiments do not exceed $10 \%$ after setting $\eta$ at 16.5 .

\section{THE SIMULATOR FRAMEWORK}

As mentioned above, the swimming and the electric models are included in a modular simulation framework based on a TCP-IP network. The highlight of this simulator is the real-time computing of the global model. This simulator computes at a frequency of $20 \mathrm{~Hz}$ (for $M \leq 1000$ ) the current state of the measurement electrodes with respect to the swimming dynamics and the surrounding environment. In accordance with Fig.4 around a TCP-IP layer, the electric fish simulator is composed of three interconnected programs: $1^{\circ}$ )

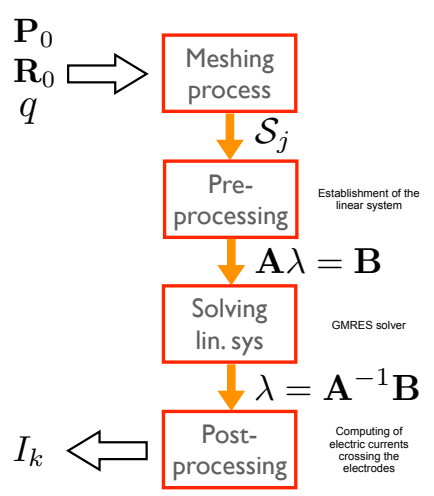

Fig. 3. Schematic view of the electric model.

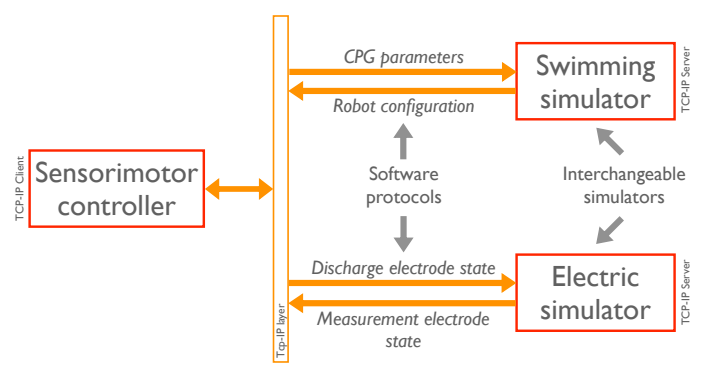

Fig. 4. Schematic view of the complete modular simulation framework.

a swimming locomotion simulator; $2^{\circ}$ ) an electric simulator; $3^{\circ}$ ) a sensorimotor controller. As regards the last point, the sensorimotor controller links perception and locomotion. It implements the obstacle avoidance behavior by using the measured currents given by the electric simulator to continuously modulate the CPG parameters used by the swimming simulator.

\section{Application to the AmphiBot}

\section{A. Electro-AmphiBot}

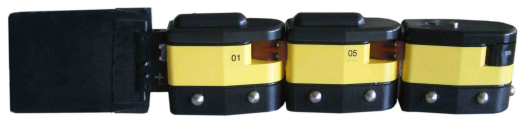

Fig. 5. The three modules AmphiBot robot equipped with the electric sense.

To illustrate the proposed multi-physic model, we address the two following problems: $1^{\circ}$ ) the study of the interactions between the body deformations and the perception variables; $2^{\circ}$ ) the design of an electric exteroceptive feedback loop for obstacle avoidance based on a direct current measurement method. In this context, we used the modular robot family of BioRob Lab as a reference. These robots named AmphiBot are fish-like robots composed by a serial assembling of identical modules which have been recently equipped with the electric sense further to a collaboration with IRCCyN $\mathrm{Lab}^{3}$ and Subatech $\mathrm{Lab}^{4}$ in the ANGELS' context (see

\footnotetext{
${ }^{3}$ http://www.irccyn.ec-nantes.fr/

${ }^{4}$ http://www-subatech.in2p3.fr/
} 
Fig.5). For this work as shown on the Fig.6, the used robot has three modules, i.e. one head $\left(m_{1}\right)$, two actuated modules $\left(m_{2}\right.$ and $\left.m_{3}\right)$ and a caudal fin. The robot has an external length of $390 \mathrm{~mm}$, a cross-section of 40 by $57 \mathrm{~mm}^{2}$, and its joint axis is placed at $20 \mathrm{~mm}$ from the front edge of segments. Moreover, the robot is neutrally buoyant, i.e. its density is equal to that of the fluid. The electric sensor is composed of five hemispherical electrodes with a diameter of $15 \mathrm{~mm}$. They are placed as follows: three on the head distributed on the left $\epsilon_{1}$, right $\epsilon_{3}$ and front $\epsilon_{2}$ of the module; two on the caudal fin, one on each lateral face $\epsilon_{4}$ and $\epsilon_{5}$. Moreover, in order to measure on each electrode an electric current, we imposed the electric potentials of $-5 \mathrm{~V}$ to the head (measurement) electrodes and $+5 \mathrm{~V}$ of the caudal fin (discharge) ones.

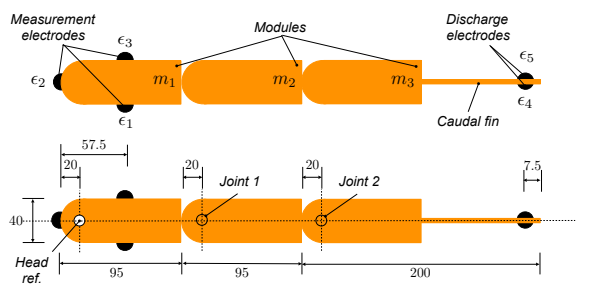

Fig. 6. The geometry of the 2-D simulated robot. All distances are in $\mathrm{mm}$.

\section{B. Interaction action-perception}

This subsection deals with the interactions between the measured currents and the body undulations (set by $\nu, A$ and $k$ ) together with the average curvature (set by $\alpha$ ). Until now, all contributions of the electro-perception in robotic (see [3], [13]) have been realized on a rigid body, i.e. the geometric configuration of the electric sensor is constant with respect to the time. Thus, the current variations, which are the inputs of perception algorithms and other controllers, are only due to the environment. Nevertheless, in this paper, the sensor is embedded in a fish-like robot. Thus, under the swimming undulation of the body shape, the sensor is deformed. As Jawad \& al have shown in [14], the currents crossing the sensor are strongly dependent of the interdistances between electrodes (see (8) of [14]), which vary in the swimming case. Hence, the geometric configuration of the sensor disturbs the measured currents which in this case is significant and hides the environment effects. In order to cancel these unwanted effects, in the following, we will propose a correction on the measured currents.

To observe the effect of the undulation on the measured currents, we carry out the following numerical test: the robot swims in a straight line towards a wall (we fix $\nu=0.8 \mathrm{~Hz}$, $A=25^{\circ}, k=0.5$ and $\alpha=0^{\circ}$ ) in a fluid with a conductivity of $0.04 \mathrm{~S} / \mathrm{m}$. As illustrated in Fig.7, we place on the robot path two insulated objects (denoted by $A$ and $B$ ) which are less conductive than the water. Hence, when the robot swims near to one of them, in accordance with the Ohm law, the local media resistivity increases and the currents through the electrodes decrease. As an illustration of the electrolocation, on Fig.8, we drew the electric field around the object $A$.

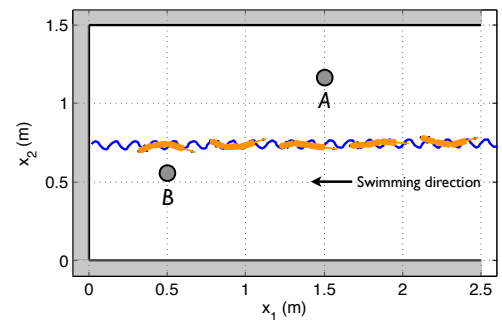

Fig. 7. The robot trajectory for the straight line swimming towards a wall test. The blue line is the trajectory of the head module.

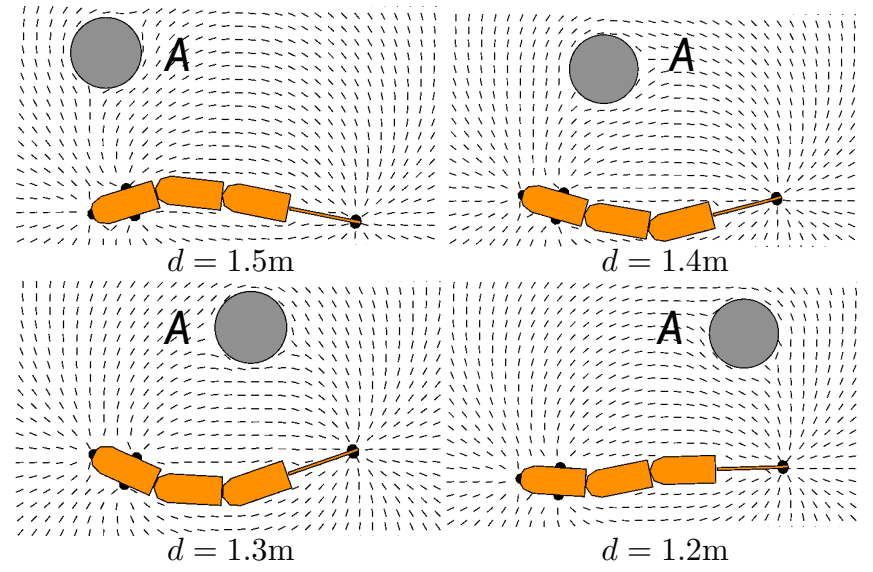

Fig. 8. The electric fields around the insulating object A. The black lines are the tangents to the electric field lines.

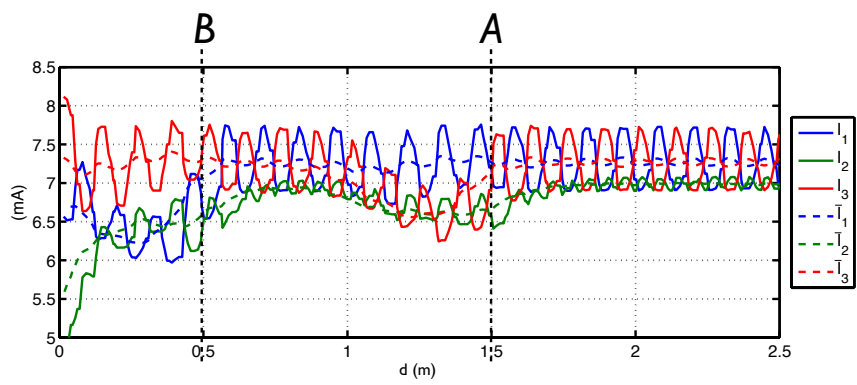

Fig. 9. The electric currents $I_{i}$ and the mean currents $\bar{I}_{i}$ measured through the electrodes $\epsilon_{i}$ in function of the wall distance $d$.

Fig.9 shows the evolution of $I_{i}$ for $i=1,2,3$ with respect to the distance $d$ between the robot nose and the wall. We observe that $I_{2}$ is disturbed by all the objects. The effect of the wall on $I_{2}$ is significant for $d \leq 0.2 \mathrm{~m}$. Near the objects $A$ and $B, I_{2}$ decreases on average of $8 \%$. On the other hand, the lateral current $I_{1}$ (respectively $I_{3}$ ) decreases on average of $15 \%$ (respectively $10 \%$ ) under the resistive effect of $B$ (respectively $A$ ) but it is not affected by the object $A$ (respectively $B$ ) and the wall. In view of these first observations, we can deduce that $\epsilon_{2}$ detects objects in front of the robot while $\epsilon_{1}$ and $\epsilon_{3}$ detect objects on the left or right respectively. As far as the current oscillations are concerned, they are due to body swimming deformations. Their effects are of the order of $7 \%$ which can conceal 
environment effects and reduce the performances of possible perception or control algorithms. Thus, in order to minimize the undulation effects on measurements, a solution will be to work with the mean currents $\bar{I}_{i}(t)=\int_{t-1 / \nu}^{t} I_{i}(t) d t$ (see dot lines in Fig. 9).

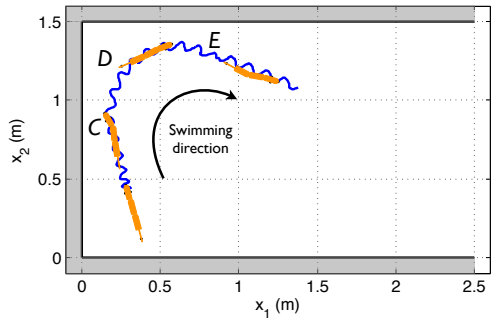

Fig. 10. The preset robot trajectory for the turning within a corner test. The blue line is the trajectory of the head module.

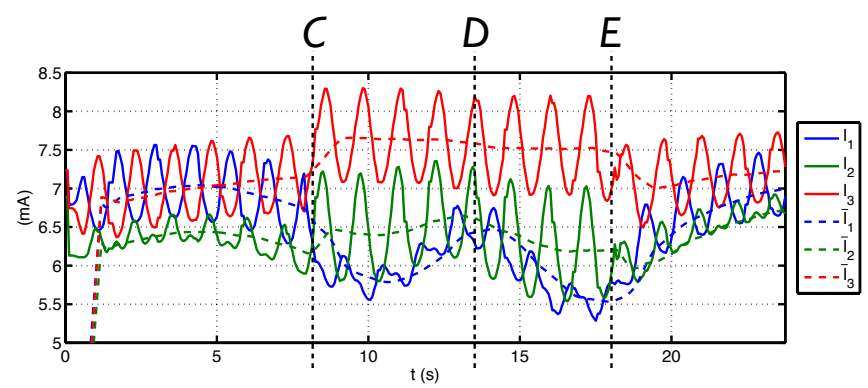

Fig. 11. The electric currents crossing the electrodes in function of the time $t$.

As the undulation effect has been highlighted, let us look at to the curvature disturbances. To illustrate them, we carry out a simulation where the robot turns within a corner. As illustrated in Fig.10, the (preset) motion path of the robot is as follows: $1^{\circ}$ ) the robot goes forward to the point $C$; $2^{\circ}$ ) between $C$ and $E$, it turns to the right with a constant curvature $\alpha=20^{\circ} ; 3^{\circ}$ ) after $E$, it goes forward again. In Fig. 11, we have plotted $I_{i}$ and $\bar{I}_{i}$ measured on the robot head during the test described above. In view of the results obtained in the straight line test, we could expect that $\bar{I}_{3}$ is constant during the turning test, but this is not the case. In fact, the disturbance observed on $\epsilon_{3}$ is due to the body curvature, which moves electrodes closer to each other. To characterize this interaction, we carried out a study in infinite space (i.e. a free environment), where we measured currents denoted by $\bar{I}_{i \infty}$ for different values of the curvature $\alpha$ (see Fig.12). In order to remove the contribution of the curvature from $\bar{I}_{i}$, for any swimming motion, we propose the following correction: $I_{i}^{c}=\bar{I}_{i}-\bar{I}_{i \infty}(\alpha)+\bar{I}_{i \infty}(0)$, where $I_{i}^{c}$ is the corrected measured current through $\epsilon_{i}$. Thus, in applying this correction to $\epsilon_{3}$ for the turning test, it is possible to reduce the curvature effect. As shown in Fig.13, the correction is efficient during the turning phase: $I_{3}^{c}$ is approximatively constant between $C$ and $E$. Let us note that the peaks observed in Fig.13 are due to the time delay introduced by computing the average. Based on these results, we designed a sensorimotor feedback loop dedicated to the obstacle avoidance.

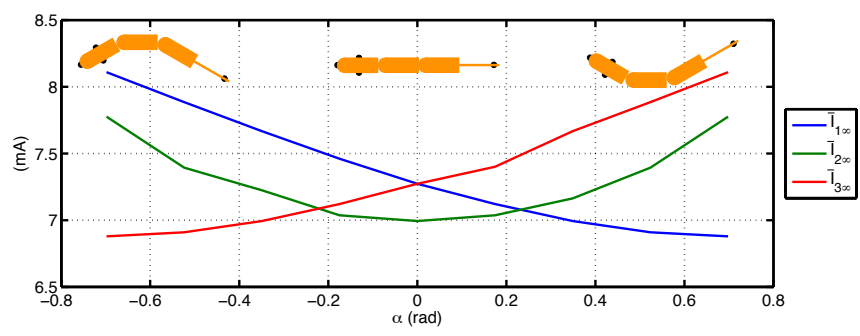

Fig. 12. The currents in infinite space with respect to $\alpha$.

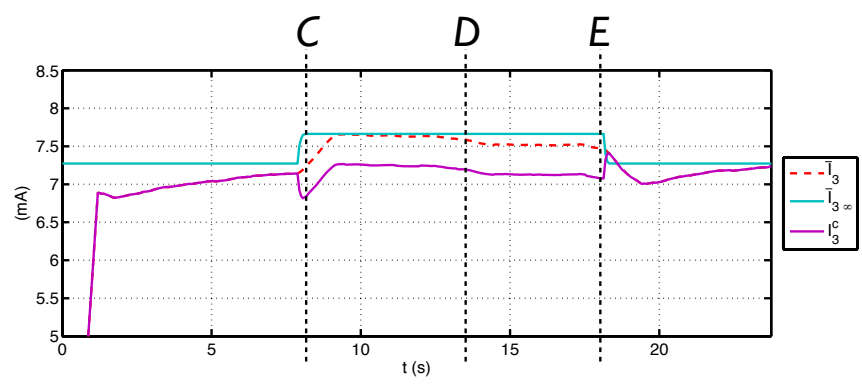

Fig. 13. $\bar{I}_{3}, \bar{I}_{3 \infty}$ and $I_{3}^{c}$ with respect to the time $t$.

\section{Application to the obstacle avoidance}

The proposed electric exteroceptive feedback does not use electric models of the environment but simple mathematic operations on $I_{i}^{c}$. In studying the signs of these variables, the low-level-perception algorithm can detect an insulating obstacle in the surrounding of the robot, on the left, on the right or in front of it and find a free area to escape. The proposed feedback loop is based on the following variables: $c_{f}=\beta_{f}\left(I_{1}^{c}-I_{3}^{c}\right)$, and $c_{h}=\beta_{h}\left(I_{2}^{c}+\bar{I}_{2 \infty}(0)\right)$, where, $c_{f}$ is the left-right difference between measured currents on the robot's head, whereas $c_{h}$ indicates the presence or not of an insulated object being in front of the robot, $\beta_{h}$ and $\beta_{f}$ are sensibility gains. Then, the idea consists, as function of $c_{f}$ and $c_{h}$, to drive the average body curvature in order that the robot turns towards a free space, by using $\alpha=\beta_{\alpha} \operatorname{atan} 2\left(c_{f}, c_{h}\right)$, where $\beta_{\alpha}$ is a sensibility gain.

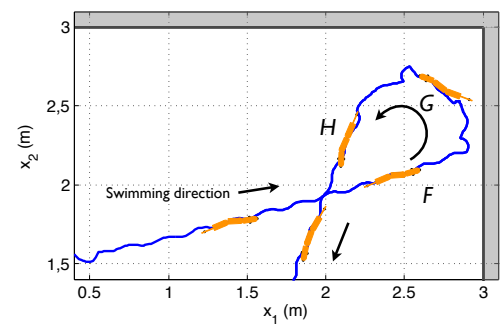

Fig. 14. The robot trajectory obtained using the control law for obstacle avoidance. The blue line is the trajectory of the head module.

In order to illustrate the working of the proposed lowperception algorithm, the first example is dedicated to corner avoidance. In this example, we present the different outputs 
of the perception and locomotion algorithms. Fig.14 shows the trajectory of the robot in the tank. This trajectory produced by the obstacle avoidance control law is composed of three parts $\left.: 1^{\circ}\right)$ start of the turn $(F) ; 2^{\circ}$ ) effect of the corner $\left.(G) ; 3^{\circ}\right)$ end of the turn $(H)$. Fig. 15 shows the mean currents and the internal variables of the control law. Fig.15 shows the time evolution of $\alpha, q_{1}$ and $q_{2}$. More precisely, on the start of turn, $I_{3}$ decreases, thus $c_{f}$ and $\alpha$ decrease while $c_{h}$ increases. Then, the robot under the effect of the feedback control law bends on the left. As regards the effect of the corner, the robot being far from the corner, $c_{h}, c_{f}$ and $\alpha$ are close to zero. The robot goes in strait line to the next wall. The wall being an insulated object and being on the right, the robot bends to the left. At the end of the turn, the robot goes in strait line to the next obstacle.
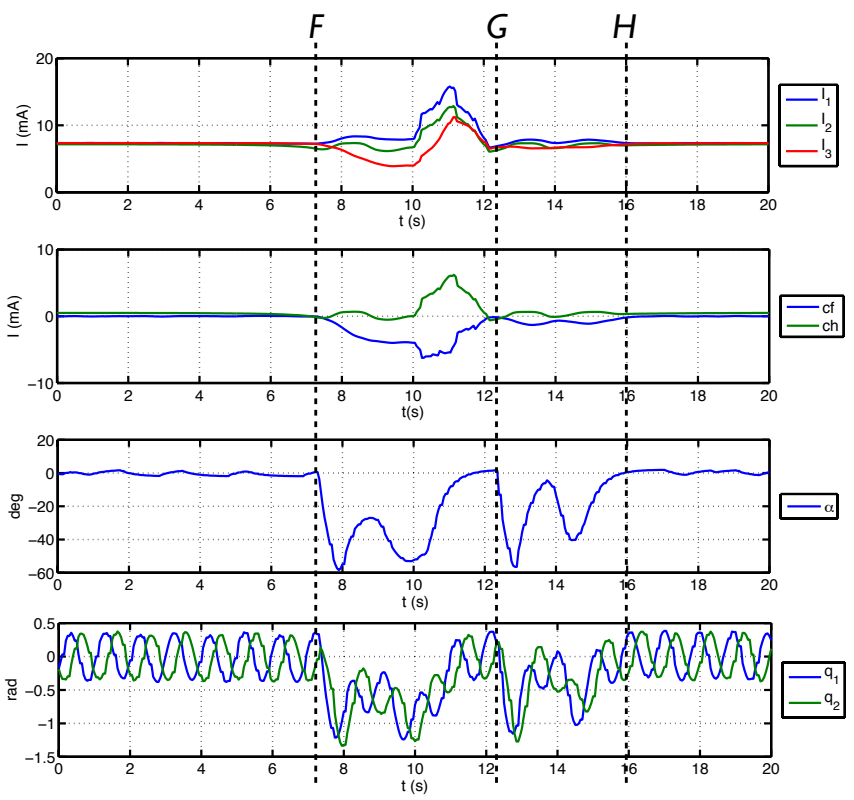

Fig. 15. $\bar{I}_{i}, c_{h}, c_{f}, \alpha, q_{1}$ and $q_{2}$ with respect to the time $t$ for the avoidance of a corner.
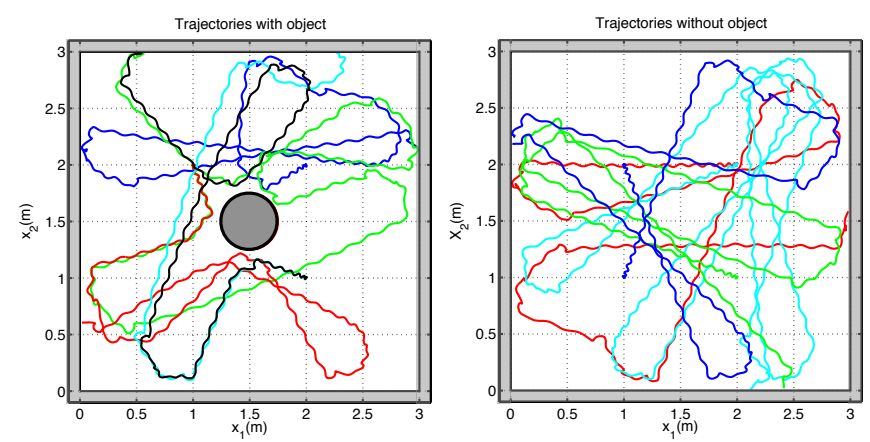

Fig. 16. Several trajectories of the robot with and without object.

In the second and last examples, two tests have been done. In the first one, the robot moves in an empty tank, while for the second one, the robot avoids an object in the same tank. Fig.16 shows 5 trajectories of the robot in the tank with and without an insulating object. The test is stopped when the head of the robot goes out of the tank which happens when an obstacle is detected too late.

\section{CONCLUSIONS}

In this paper, we have presented some results regarding sensorimotor feedback in the case of an electric fish-like robot. For this work, we have designed a multi-physics simulator working in real time. This simulator solves in parallel the swimming locomotion problem and the electrokinetic equations. Using this tool, we studied the interactions between the currents crossing the electrodes and the body shape. On this base, we proposed a current correction process in order to cancel the unwanted effects of the anguilliform swimming on the perception. Thanks to these results, we designed a low-level-perception algorithm to address the obstacle avoidance problem of an electric fish-like robot. With the proposed controller based only on 3 measurements, the robot can swim safely for a quite long duration.

\section{ACKNOWLEDGMENTS}

The ANGELS project is funded by the European Commission, Information Society and Media, Future and Emerging Technologies, contract number: 231845. Finally, the authors gratefully acknowledge Pol-Bernard Gossiaux for rewarding exchanges on electromagnetism.

\section{REFERENCES}

[1] H. Lissmann and K. Machin, "The mechanism of object location in gymnarchus niloticus and similar fish," The J. of Experimental Biology, no. 35 , pp. 451-486, 1958.

[2] B.Rasnow, "The effects of simple objects on the electric field of apteronotus," J. of Comparative Physiology A, vol. 3, no. 178, pp. 397-411, 1996.

[3] J. Solberg, K. Lynch, and M. MacIver, "Active electrolocation for underwater target localization," Inter. J. of Robotics Research, vol. 27, no. 5, pp. 529-548, 2008.

[4] A. Crespi and A. Ijspeert, "Online optimization of swimming and crawling in an amphibious snake robot," IEEE Trans. on Robotics, vol. 24, pp. 75-87, 2008.

[5] W. Khalil, G. Gallot, and F. Boyer, "Dynamic modeling and simulation of 3-d serial eel-like robot," IEEE Trans. Systems, Man and Cybernetics - Part C: Applications and reviews, vol. 37, pp. 1259-1268, 2007.

[6] F. Boyer, M. Porez, A. Leroyer, and M. Visonneau, "Fast dynamics of an eel-like robot - comparisons with navier-stokes simulations," IEEE Trans. on Robotics, vol. 24, no. 6, pp. 1274-1288, 2008.

[7] F. Boyer, M. Porez, and A. Leroyer, "Poincaré-cosserat equations for the lighthill three-dimensional large amplitude elongated body theory: application to robotics," J. of Nonlinear Science, vol. 20, pp. 47-79, 2010.

[8] C. M. Breder, "The locomotion of fishes," Zoologica, vol. 4, pp. 159256, 1926.

[9] J. Lighthill, "Large-amplitude elongated body theory of fish locomotion," Proc. of the Royal Society of London, Series B, vol. 179, pp. 125-138, 1971.

[10] M. M. Munk, "The aerodynamic forces on airship hulls," NACA, vol. Rep. 184, 1924.

[11] G. I. Taylor, "Analysis of the swimming of long narrow animals," Proc. of the Royal Society of London, Series A, vol. 214, pp. 158-183, 1952.

[12] L. Gaul, M. Kögl, and M. Wagner, Boundary element methods for engineers and scientists. Springer-Verlag, 2003.

[13] V. Lebastard, C. Chevallereau, A. Amrouche, B. Jawad, and al, "Underwater robot navigation around a sphere using electrolocation sense and kalman filter," in IEEE IROS, 2010.

[14] B. Jawad, P. Gossiaux, F. Boyer, V. Lebastard, and al, "Sensor model for the navigation of underwater vehicles by the electric sense," in IEEE ROBIO, 2010. 